\title{
Corrigendum to "Collaborative Computing and Resource Allocation for LEO Satellite-Assisted Internet of Things"
}

\author{
Tao Leng, ${ }^{1,2}$ Xiaoyao Li, ${ }^{1,2}$ Dongwei Hu, ${ }^{3}$ Gaofeng Cui $\mathbb{D}^{1,2,3}$ and Weidong Wang ${ }^{1,2}$ \\ ${ }^{1}$ School of Electronic Engineering, Beijing University of Posts and Telecommunications, Beijing 100876, China \\ ${ }^{2}$ Key Laboratory of Universal Wireless Communications, Ministry of Education, Beijing University of Posts \\ and Telecommunications, Beijing 100876, China \\ ${ }^{3}$ Science and Technology on Information Transmission and Dissemination in Communication Networks Laboratory, 54th Research \\ Institute of China Electronics Technology Group Corporation, Shijiazhuang, China \\ Correspondence should be addressed to Gaofeng Cui; cuigaofeng@bupt.edu.cn
}

Received 6 December 2021; Accepted 6 December 2021; Published 23 December 2021

Copyright (c) 2021 Tao Leng et al. This is an open access article distributed under the Creative Commons Attribution License, which permits unrestricted use, distribution, and reproduction in any medium, provided the original work is properly cited.

In the article titled "Collaborative Computing and Resource Allocation for LEO Satellite-Assisted Internet of Things" [1], a statement noting the equal contribution of authors

Tao Leng and Xiaoyao Li was omitted in error.

\section{Authors' Contributions}

Tao Leng and Xiaoyao Li contributed equally to this work.

\section{References}

[1] T. Leng, X. Li, D. Hu, G. Cui, and W. Wang, "Collaborative computing and resource allocation for LEO satellite-assisted internet of things," Wireless Communications and Mobile Computing, vol. 2021, Article ID 4212548, 12 pages, 2021. 